\title{
SALT/HET Cooperation in Education and Public Outreach
}

\author{
Mary Kay Hemenway and Sandra Preston \\ Department of Astronomy, University of Texas, Austin, Texas \\ 78712-1083, U.S.A. e-mail: marykay@astro.as.utexas.edu
}

\begin{abstract}
The "Science with SALT" meeting in March 1998 opened avenues of cooperation between SAAO and the University of Texas at Austin in education and public outreach. This paper will review past interactions and future plans. SAAO personnel have visited the HET and McDonald Observatory and have taken part in planning meetings for the Texas Astronomy Education Center museum area and educational programming. Discussions concerning the extension of the daily radio show StarDate (English), Universo (Spanish) and Sternzeit (German) versions to a southern hemisphere version are underway. In addition, we are cooperatively planning a workshop to discuss an international collaborative for educational outreach for state-of-the-art telescopes for which a regional collaborative in southwestern U.S. (SCOPE) serves as a model. The towns of Sutherland and Fort Davis are discussing forming a "twin-town" relationship. Projects and plans that link cutting-edge astronomical research to classrooms and the public will be reviewed.
\end{abstract}

\section{Introduction}

With plans for the Southern African Large Telescope (SALT is currently under construction at Sutherland, South Africa) largely based on the design of the Hobby-Eberly Telescope (HET is located at McDonald Observatory in Texas, U.S.A.), there has been significant technology transfer between the observatories. Both observatories share another goal beyond exploring the secrets of the universe with their unique telescopes. Both are committed to providing a motivating education and public outreach program to visitors and to students. Astronomy offers unique opportunities to motivate students to learn science, mathematics, and modern technology. Their fascination with the cosmos can form the basis for a robust educational and public outreach program that reaches far beyond the physical sites of the observatories to include a much wider population.

The unique design of HET and SALT provides an opportunity to capitalize on the technology as well as the science as topics. HET has 91 interchangeable one-meter spherical mirrors arranged to form a 10x11 meter hexagon. The 
telescope is permanently tilted with azimuthal motion allowable only between observing periods. This design allows the cost to be significantly lower than for a fully-steerable optical telescope of similar size and still allows about $70 \%$ of the sky to be accessed. Celestial objects are tracked as Earth's rotation carries them across the field of view. The telescope is designed to be especially efficient for spectroscopy. Eventually, several spectrographs will be available at different resolutions. HET is mostly operated in queue-scheduled mode. Resident astronomers can change the computer schedule queue for real-time observations.

\section{Education and Public Outreach Interactions}

As engineers and scientists, and lawyers, planned the cooperative agreements that exchange observing time for technical plans and assistance, the scientists and staff especially interested in Education and Public Outreach (EPO) at these observatories began to exchange visits and information (without the lawyers). The first major meeting took place at the "Science with SALT" conference at the South Africa Astronomical Observatory (SAAO) in March 1998. A significant portion of the meeting was devoted to increasing science awareness and providing educational opportunities. Formal presentations and focus groups involving many of the participants provided for a diverse and broad exchange of information. (Hemenway 1998; Preston 1998; Rijsdijk 1998)

Following that meeting, Case Rijsdijk of SAAO was invited to participate in the national Advisory Board meeting for the "Decoding Starlight" exhibit plans in May 1998, in Fort Davis, Texas. This exhibit will form a principle part of the new Texas Astronomy Education Center (TAEC). The 11,000 square-foot building with its theater, exhibit hall, classroom, and visitor amenities will be the focal point for outreach at McDonald Observatory. An outdoor observing area and telescope park adjoin the TAEC.

In July 2000, Frans Hanekom, Mayor of Sutherland, Alleta van Sittert, Town Clerk of Sutherland, and Peter Martinez of SAAO came to Fort Davis to participate in the TAEC groundbreaking ceremonies. They reviewed plans, toured current visitor facilities, and made arrangements for a Twin Town agreement between Sutherland and Fort Davis. In September 2000, Preston and Judge Peggy Robertson of Fort Davis participated in the groundbreaking of SALT and the signing of the Twin Town agreement.

Plans for a major meeting of EPO personnel of the world's largest observatories are underway. The Large Telescope Educational Collaboration (LATEC) Workshop will take place at the SAAO in February 2001. LATEC may be modeled on SCOPE (the Southwestern Consortium of Observatories for Public Education; see: http://hyperion.as.utexas.edu/mcdonald/scope/). By forming a partnership of observatories, we will be able to create a cooperative spirit, limit duplication of effort, leverage funds, provide a support network in a career arena where there are only a handful of institutions, create opportunities for professional development, and share challenges and solutions. 


\section{The Texas Astronomy Education Center}

The TAEC is more than a building and outdoor observing facility. It is part of a coordinated program to bring astronomy research to the public and to teachers. The TAEC theater will offer presentations to the public and the classroom will be used by visiting school groups and for teacher workshops; the remoteness of the observatory does not restrict access to astronomy to only those who can travel to the TAEC. A visit of a few hours can do little to fill in what is often rather poor background in astronomy. The goal of the TAEC is to use the beauty and excitement of astronomy as the motivator to increase science literacy at all levels. The Internet is a powerful tool that can be used to deliver pre- and post-visit information and activities to teachers, and to serve as an informative and exciting resource for the public and educators. The activities will also be delivered through workshops at teachers' meetings and within school districts.

The exhibits and workshops have a special emphasis: spectroscopy. Preparing museum exhibits demands special care. Rowan (1992) noted "An idea may be confusing because it involves (a) difficult language, (b) difficult-to-picture structures or processes or (c) difficult-to-believe notions (e.g. Earth is weightless)." In surveys conducted with McDonald Observatory visitors, we found that most people are unfamiliar with spectroscopy and, in fact, they stumble over the word the first time they try to say it. While most visitors know astronomers use telescopes to study the universe, they believe telescopes are used to collect "information." Many people don't know telescopes are collecting light or that astronomers are analyzing light -light that has been traveling to us for billions of years - to learn about the Universe. Feher and Rice (1985) identified two important characteristics of museum exhibitions about light that appeared to greatly improve their ability to produce a full understanding of physical phenomena. These characteristics are (1) that an exhibit is usable by the visitor in different ways, offering more varied exploration of a topic, and (2) that different exhibits explore the different aspects of the same phenomenon, providing a "richer learning atmosphere." For these reasons, the exhibits begin with the key ideas that "sunlight is starlight" and the nature of light and spectra. All the exhibits have captions and keys in both English and Spanish. The variety of exhibits allows visitors to explore topics though different media - reading, observing, interacting, and experimenting - that are suitable for different levels of understanding. For example, some exhibits are aimed at rather young children and others allow those with prior knowledge of chemistry and physics to probe deeper their applications in learning about the composition, evolution, and motions of celestial objects.

In preparation for the opening of the TAEC, Hemenway and Armosky (2000) have developed and field-tested a new series of instructional units aimed at teachers. Standard ways of teaching about the nature of telescopes must be augmented to explore the innovative technology used in HET and SALT. "Telescope Technology for Teachers" allow students to explore the construction, pricing, and structural design of HET. Students use small inexpensive flat mirrors to build a human model of the HET; the students serve as the mirror actuators that tilt, tip or piston the individual segments into position and then they aim a flashlight beam at a target. Their success is measured (as it is for the actual telescope) by how small a target they can hit and how long they can 
hold it steady. Other activities illustrate the use of the tracker and fiber optic. Armosky and Hemenway are preparing a parallel set of "hands-on, minds-on" activities that explore spectroscopy. All materials are aligned with the National Science Education Standards (National Research Council 1996).

Just as SAAO has posted a series of their activities on the Internet (http://www.saao.ac.za/education/modules.html) to share with the world, these McDonald Observatory activities will be available on a special web page (http://stardate.utexas.edu/marykay/ttt/ttt.html). SALT and HET are already freely sharing their educational activities with each other.

Among the other resources produced at McDonald Observatory are the daily radio show StarDate (in English, with its companion programs in German "Sternzeit" and Spanish "Universo"). (Barnes, 1996) Six issues per year of StarDate magazine provide another avenue for the public to learn about astronomy. Special publications such as a guide to the solar system (McDonald Observatory, 1998a,b) in English and Spanish and educational posters with classroom activities are available for teachers.

\section{Twin Towns}

The twin town agreement of 1 September 2000 unites the similarly-sized, remote, rural communities of Fort Davis and Sutherland (respective populations of 800 and 2000) to explore the ways that will enable them to serve the increasing numbers of visitors drawn by the existence of the major astronomical facilities nearby. Both towns have immediate infrastructure developmental problems that need addressing over an extended period. The agreement is meant to be the beginning of a process of sharing information, expertise, and friendship between the communities. As part of the agreement, communications between local schools and individual children will help both explore the cultural dynamics of their communities. The private sector members will explore business practices, retailing products, marketing strategies and small business entrepreneurship as they discuss the changes that occur as a rural entity becomes a tourist destination.

Among the immediate plans are to exchange exhibits, photos, maps, and respective flags. The schools will identify projects to share. Individual children will be linked by mail or electronic mail to other children. Individual businesses will be encouraged to link with each other, and eventually exchange of retail products may take place. Websites will be created for each town to list business/tourism opportunities and historical backgrounds.

Cooperation between SALT and HET has led to an opportunity to broaden the cultural and economic life of two widely separated communities.

\section{Conclusion}

By working together in these efforts, we will be able to create a cooperative spirit, limit duplication of effort, leverage funds, create opportunities for professional development, and share challenges and solutions. The unique features of our similar telescopes bring us close together. Although each country can claim particular features of its educational system, we share similar problems. We each deal with a bilingual or multi-lingual population. We each want to use 
astronomy to inspire and motivate students and young people into considering careers in science and/or engineering. And, we each wish to increase the general science literacy level of our citizens. SALT and HET have provided us with an arena in which to work together.

\section{References}

Barnes, S. P. 1996, UNIVERSO: A Spanish-Language Astronomy Radio Program in Astronomy Education: Current Developments, Future Coordination, ASP Conference Series vol. 89, J. R. Percy, ed., 165-166.

Feher, E. and Rice, K. 1985, Development of Scientific Concepts through the Use of Interactive Exhibits in A Museum. Curator, 28, 35-46.

Hemenway, M. K. 1998, Building Public Support for Astronomy though School Based Education in Science with SALT: proceedings of the SALT/HET Workshop. D.A.H. Buckley, ed. 157-163.

Hemenway, M. K. and Armosky, B. J. 2000, Telescope Technology for Teachers. Bulletin of the American Astronomical Society, 32, 1559.

McDonald Observatory, 1998a, StarDate Guide to the Solar System.

McDonald Observatory 1998b, Universo Guia Del Sistema Solar.

National Research Council 1996, National Science Education Standards (Washington, DC).

Preston, S. 1998, Astronomy for the Masses: Making Research Relevant for Everyone in Science with SALT: proceedings of the SALT/HET Workshop. D.A.H. Buckley, ed. 151-155.

Rijsdijk, C. 1998, Friends with the Universe: Taking Astronomy to the People in Science with SALT: proceedings of the SALT/HET Workshop. D.A.H. Buckley, ed. 165-171.

Rowan, K. E. 1992, What Research Says: No. 19 About Explaining Diffcult Ideas. Association of Science and Technology Centers Newsletter, Nov./Dec. 1992, 7-8, 10. 